\title{
Le banquet de Rachilde
}

Vicky GAUTHIER, Université du Québec à Chicoutimi

L'amour divin à une époque où il n'y a plus de dieu. L'amour qui brûle à une époque où le monde se refroidit.

Rachilde, La jongleuse

Au fil de mes recherches dans le cadre de ma thèse portant sur l'œuvre romanesque de l'écrivaine française Rachilde (1860-1953), il m'a semblé que l'ensemble de ses personnages, tous plus anormaux les uns que les autres, ne parle que d'une chose : d'amour. Mais quel est-il précisément? Quelle est sa nature ? Ses caractéristiques ? Y a-t-il une cohérence, un fil d'Ariane qui unirait les romans de Rachilde, permettant d'y dégager une philosophie de l'amour qui serait propre à l'auteure ? Dans cet article, je vous invite à plonger avec moi dans l'univers étrange et décadent de Rachilde afin de s'intéresser à la façon dont celle-ci s'occupe des choses de l'amour et le conçoit. D'emblée, le titre de l'article annonce une certaine filiation philosophique avec Platon. Je vous propose donc, à l'instar du célèbre Banquet platonicien (sur le plan de la forme et parfois du contenu), un éloge de l'amour, ici en trois temps, à travers la prose rachildienne ${ }^{1}$. Pour ce faire, je ferai appel à quelques romans-clés de l'écrivaine où un discours philosophique sur l'amour est élaboré, et à un texte pamphlétaire au titre et au contenu provocateurs (qui lui a d'ailleurs valu une forte condamnation de la part de ses pairs et qui l'a entraînée du côté obscur/oublié de l'histoire littéraire), Pourquoi je ne suis pas féministe (1928).

\section{Antiquité et mythologie : un amour du passé}

L'esprit fin-de-siècle abhorre le présent et tout ce qu'il contient de modernités. Bon nombre de personnages rachildiens, en particulier ceux figurant dans les romans parus au courant du vingtième siècle ${ }^{2}$, se positionnent fortement contre tout ce qui est moderne et se languissent d'une époque plus simple, primitive et glorieuse. Leur entourage mentionne très souvent leur appartenance à un autre monde, à un autre temps, à un ailleurs n'allant pas de pair avec le présent, sorte d'anomalie à la fois étrange et irrésistible. Par exemple, dans Madame de Lydone, assassin, on dit du personnage éponyme qu'elle «était hors du temps, des conventions et de l'existence normale » (18). Tandis que dans La jongleuse, la veuve Éliante Donalger est décrite comme « à la fois très chez elle et très en dehors de tous les mondes » (37). Ce faisant, leur 
conception de l'amour ne peut être contemporaine : «très seul[s] de [leur] espèce » (Madame de Lydone, 25), ceux-ci «n'entend[ent] pas la passion ordinaire » (La jongleuse, 85) et se tournent vers d'autres pratiques amoureuses, hors normes de surcroit ${ }^{3}$, tout en vouant un culte à l'Amour/la Volupté à travers diverses divinités et autres mythes anciens aux héros légendaires : Éros, Narcisse, Orphée, Antinoüs ${ }^{4}$, Pygmalion, etc. Raoule de Vénérande, dans Monsieur Vénus, convoque l'Antiquité pour expliquer sa «passion contre nature »(74), tout en critiquant, au passage, sa propre époque :

Dans l'antiquité, [...] le vice était sacré parce qu'on était fort. Dans notre siècle, il est honteux, parce qu'il naît de nos épuisements. Si on était fort, et si de plus on avait des griefs contre la vertu, il serait permis d'être vicieux [...]. (73)

Oublions la loi naturelle, déchirons le pacte de procréation, nions la subordination des sexes, alors nous comprendrons les débordements inouïs de cette autre prostituée qui fut l'antiquité païenne. Quelle passion aujourd'hui qualifiée vice ou monstruosité ne fut pas alors chantée, encensée, déifiée. L’Olympe est peuplé de dieux bâtards qui tous eurent leurs poètes, leurs adeptes, leurs sacrificateurs. (9293)

De même, l'espace intime dans les romans rachildiens, chargé d'objets à l'effigie de ces figures antiques ou leur faisant référence, constitue un «temple» (La jongleuse, 110 ; Nono, 173 ; Madame Adonis, 193), un « sanctuaire ${ }^{5}$ » (La jongleuse, 111) au sein duquel ces pratiques ont lieu. Le décor chez Rachilde est à considérer comme un signe annonçant l'inclination amoureuse/érotique du ou de la propriétaire des lieux pour ces dieux et mythes (on y retrouve, entre autres, statues et/ou statuettes, lit sculpté, toiles représentant des Amours, des éphèbes, Éros, Antinoüs, etc.). Pour ce qui est de la représentation de la femme à cette époque - fortement misogyne, rappelons-le -, les choix demeurent limités: «la bête ridicule ou la fatale perversité $^{6} »$, nous dit Nathalie Prince dans son ouvrage Les célibataires du fantastique (2002), ce qui explique la profusion de figures mythologiques à connotation négative, «telles Salomé, Hérodiade ou Astarté, [sans oublier Méduse et Pandore] ${ }^{7} »(46)$. Toutefois, ce qui surprend chez Rachilde est la présence dans certains de ses romans de la déesse romaine de la chasse, Diane ${ }^{8}$. Sœur d'Apollon, née quelques instants avant lui, elle est témoin des douleurs de l'enfantement et rejette alors violemment le mariage (et choisit donc de rester vierge). Diane semble être une figure toute désignée pour la Décadence, en tant que femme célibataire. Pourtant, elle n'apparaît nulle part dans les ouvrages dédiés à la littérature de cette période : Evanghélia Stead, dans son important livre sur les figures de la Décadence, Le monstre, le singe et le foetus. Tératogonie et 
Décadence dans l'Europe fin-de-siècle (2004), ne fait aucunement mention de la déesse Diane. Quant à Prince, elle omet non seulement la déesse lorsqu'il est question des figures antiques auxquelles se rattache la Décadence, mais rejette aussi l'existence de tout personnage célibataire féminin (qui serait autre chose que simple objet de désir et/ou de mort ${ }^{9}$ ). Néanmoins, on ne peut nier la présence de ces femmes célibataires des œuvres de Rachilde ni leur volonté de vivre tout aussi librement que le célibataire mâle. Par exemple, Mary Barbe, dans La marquise de Sade, évoque les mêmes raisons que la déesse Diane concernant son refus de procréation : "Ma mère est morte là, Monsieur, en mettant mon frère au monde ; moi je ne veux pas mourir de la même manière, et, en supposant que je ne meure pas... je ne veux pas subir la torture d'un accouchement »; «Je vous dis cyniquement : je ne veux pas être mère, d'abord parce que je ne veux pas souffrir, ensuite parce que je ne veux pas faire souffrir. C'est mon droit [...]. Je ne connais pas de puissance humaine capable de me faire fléchir» $\left(214 ; 214-215^{10}\right)$. Par ailleurs, on trouve dans d'autres romans des statues de la déesse chasseresse (Nono, La virginité de Diane $^{11}$ ), de même qu'une description physique du personnage féminin faisant référence à Diane (Monsieur Vénus, La marquise de Sade, Madame Adonis ${ }^{12}$ ), sans compter toutes celles qui refusent le mariage (L'Amazone rouge, L'Animale) ou bien le remariage (La jongleuse, Madame $A_{d o n i{ }^{13}}{ }^{13}$ - à la suite du décès de l'époux -, faisant aussi directement écho à Diane et à son choix délibéré de célibat. Comme premier élément de l'amour rachildien, il y a bel et bien cette allégeance à un passé antique révolu dans la façon d'envisager l'amour, sorte de langueur pour une époque moins complexe et surtout plus libre. Passons maintenant au deuxième discours.

\section{À la recherche de cette moitié perdue ou le mythe de l'androgyne}

Le discours d'Aristophane dans Le banquet de Platon est très certainement le plus connu de l'ouvrage. Ce dernier raconte l'origine de l'amour à travers le mythe de la création, où Zeus fâché contre les humains pour leur orgueil et leur insolence - les a séparés et dispersés afin de réduire leur force ${ }^{14}$, puisqu'ils formaient auparavant un tout avec leur âme sœur, genre de monstre à une tête, mais à deux visages, et à quatre mains et jambes, soit mâle, femelle ou androgyne (70). Ainsi, l'amour est, selon Aristophane, le « désir et [...] la recherche de cette nature d'une seule pièce », de cette complétude (79). Rachilde et les décadents reprennent ce mythe et s'en nourrissent, bien entendu, de façon détournée. C'est dans Monsieur Vénus que l'on trouve le mythe de l'androgyne relaté par Aristophane ${ }^{15}$. La description de la valse de Raoule et 
Jacques lui fait directement écho et le subvertit (sous forme de chiasme en reversant les genres sexuels). Raoule, qui est originellement une femme, se travestit, se masculinise au contact de Jacques, qui, lui, se féminise :

Il valsait bien ce manant [Jacques], et son corps souple, aux ondulations féminines, semblait moulé pour cet exercice gracieux. Il ne cherchait pas à soutenir sa danseuse [Raoule], mais il ne formait avec elle, qu'une taille, qu'un buste, qu'un être. À les voir pressés, tournoyants et fondus dans une étreinte ou [sic] les chairs, malgré leurs vêtements, se collaient aux chairs, on s'imaginait la seule divinité de l'amour en deux personnes, l'individu complet dont parlent les récits fabuleux des Brahmanes, deux sexes distincts en un unique monstre. (156-157)

Le roman Refaire l'amour, à travers Alain de Montarès, narrateur homodiégétique du récit, fait aussi état de cette recherche de l'autre, cette moitié perdue : «Je crois que le couple erre à travers les siècles en se cherchant et qu'il ne se reforme que par un miracle des circonstances. Et c'est sans doute pour cela qu'on n'aime qu'une fois en toute connaissance de cause. [...] La puissance éternelle c'est l'amour » (135-136). Dans son pamphlet Pourquoi je ne suis pas féministe, Rachilde consacre un chapitre complet à l'amour ; certains propos rappellent tantôt ceux d'Aristophane, tantôt ceux de Socrate. En voici trois extraits : «L'amour, cette électricité, cette étincelle d'une flamme d'origine inconnue, cherche, tout naturellement, $l a$ flamme sœur... et ne la rencontre presque jamais »; «L'amour n'est pas seulement un sentiment pour quelqu'un, c'est l'épanouissement d'un cerveau touché par lui et l'objet n'est plus en question. On peut avoir de l'amour pour n'importe qui et cela suffit à mener au paroxysme de toutes les énergies l'être victime de cette... illumination»; «Tous les hommes et toutes les femmes sont-ils capables de brûler de cette flamme? Non. De même qu'un microbe ne peut se développer dans certaines organisations, l'amour ne peut pas intoxiquer tout le monde. Cependant il est à remarquer que ce sont les plus riches cerveaux, les plus beaux tempéraments qui peuvent être atteints de ce que je me permettrai d'appeler : la céleste maladie» (48-49). Ainsi, nous avons ici les bases de l'amour rachildien : il se rattache à quelque chose d'ancien ; il ne vise ni le mariage ni la procréation et il aspire à la complétude (quoiqu'elle ne se réalise que très rarement). Passons maintenant au troisième et dernier discours rachildien sur l'amour.

\section{Le culte de la beauté}

Dans la même ligne de pensée, ce qui ressort le plus dans les romans de Rachilde ainsi que dans son pamphlet Pourquoi je ne suis pas féministe est l'idée d'un amour supérieur, 
dépassant le monde humain ordinaire, trivial et éphémère, au profit d'un amour plus sublime, idéel et éternel :

C'est certainement le souffle d'un dieu inconnu qui disperse la raison ou allume une flamme très en dehors de nos foyers humains. Je ne parle pas du vulgaire attrait qu'un sexe a pour l'autre : ceci est du ressort purement (ou impurement) humain et cela n'a rien à voir avec l'amour. [...] L'un n'empêche pas l'autre, mais il y a très souvent des cas ou [sic] l'autre domine et finit par anéantir la cause... commune. Je n'ai pas du tout la prétention de vous imposer ma manière de voir, cependant elle est encore la seule façon d'expliquer... l'inexplicable. (49)

Cette hiérarchie amoureuse évoque celle qu'offre Platon à travers le discours de Socrate dans Le banquet, à savoir que l'amour doit sans cesse chercher à s'élever des choses terrestres (corps) à «l'essence même du Beau ${ }^{16} »$ (âme). Pour Rachilde, l'amour doit aussi dépasser ce simple besoin physique de reproduction (sans cesse dénigré par l'écrivaine) au profit d'un amour plus cérébral, éthéré, la menant à dire, toujours dans son pamphlet, que « Le grand amour est calme, très au-dessus des agitations fébriles, de toutes les mondanités » (50-51). Dans Le grand saigneur, c'est le marquis Yves de Pontcroix qui nous fait part de cet amour supérieur, qui n'est pas de ce monde :

$[\mathrm{M}]$ on amour, à moi, est d'une essence plus rare que celui des humains, parce qu'il ne finit pas. La satiété ne le menace pas. Il n'a pas le but ridicule de la procréation. Il faut laisser ce soin à nos domestiques. La procréation est un usage de basse-cour ou d'étable, et elle fournit assez d'esclaves pour que les gens libres ne s'en occupent pas. [...] Une chose compte, puisque c'est sur cela que repose la loi de la vie : la volupté. Or, la volupté n'est belle qu'à l'état pur. (270-271)

Autre type d'amour, donc autre langage, il s'agit bel et bien ici, comme nous l'indique le titre du roman de Rachilde, de Refaire l'amour ${ }^{17}$, de le réinventer. Dans La jongleuse, lorsque la veuve Éliante tente d'expliquer sa conception de l'amour à son jeune disciple, Léon Reille, elle lui dit: «C'est vrai que nous parlons [sic] pas encore la même langue ? Cela viendra ! (75). Pontcroix souhaite aussi convertir sa fiancée à cet amour nouveau genre : «Marie, je suis heureux, mais je suis impuissant à vous plaire, parce que je ne sais pas me plier à l'amour humain. [...] Et tu attends de moi l'amour humain »; «Je ne suis pas plus fou que ceux qui songent à l'amour tel qu'on le parle en votre langue humaine ». Et il termine son propos en murmurant ceci : «Quand je pense que je pourrai peut-être la [Marie] convertir à ma religion ! » (Le grand saigneur, 194-195 ; 197). Cette idée de pureté et d'éternité - tout à fait platonicienne revient constamment dans l'œuvre romanesque de Rachilde. Chez Platon, l'immortalité passe, quand il est question du corps, par la reproduction de l'espèce, mais aussi par l'Art, quand il est 
question de l'âme (125-126). Cela va sans dire que chez Rachilde et les décadents, l'immortalité se rattachera plutôt à l'Art, à la Beauté, à la Volupté, qui constituent une véritable doctrine amoureuse.

Perçus comme étranges et «en dehors de toute la société » (L'animale, 151) pour qui les côtoie, les personnages rachildiens se posent donc en représentants de cet Amour autre, en tant qu'envoyé(e) ou personnification de Dieu, de l'Amour, de la Volupté. L'exemple le plus notable est Éliante Donalger dans La jongleuse. S'autoproclamant " prêtresse » et affirmant qu'elle est « habitée par un dieu », qu'elle «est l'Amour » même $(84 ; 150-151)$, Éliante est une philosophe de l'Amour, dont le discours s'apparente à celui de Socrate dans Le banquet. Il s'agit du personnage jetant le plus clairement les bases de l'amour rachildien dans l'ensemble de son œuvre, que suivent plus instinctivement les autres personnages qui ne sont pas aussi conscients et loquaces qu'elle. En effet, tout comme Socrate pour qui l'amour est ce qui nous élève vers le Beau, le Bon, en passant du tangible (corps) au sublime (âme), l'Éliante de Rachilde apparaît comme une maîtresse (dans le sens d'enseigner) de l'Amour s'étant détournée de tout amour normal pour être « réellement amoureuse de tout ce qui est beau, bon, [qui lui] paraît un absolu, la définition même de la volupté » (49) et ayant « le désir [...] de faire du bien, de faire plaisir, de communiquer [s]a chaleur [...] pour inspirer le goût du beau » (167). «Recluse libre, une sorte de religieuse émancipée, une prêtresse laïque [, Éliante] ne veu[t] que convertir [Léon] à [s]a religion, qui est l'unique »(84); elle voit en lui un disciple potentiel à qui inculquer cet Amour :

Pourquoi me vulgariserais-je ?... si je n'ai pas besoin de vous. C'est vous qui devez vous élever jusqu'à moi si vous me désirez ${ }^{18}$. J'ai cru un moment que j'élèverais un homme dans le sentiment de mon genre de beauté, qu'il me viendrait un enfant vraiment né de mon amour et semblable à moi. Que je pourrais perpétuer la folie du plaisir... jusqu'à en faire du bonheur permis par les foules. Allons donc ! Les dieux sont seuls, et quand ils se promènent, par hasard, sur la terre, ce sont des cas pathologiques ou des baladins, des histrions...qu'on méprise ! (168)

La suite du roman montre en effet un monde qui n'est pas prêt de changer. S'agissant ainsi d'un amour pour le moins idéal, rares sont les romans de Rachilde qui arrivent à mettre les paroles en actes, puisque la nature humaine - toujours intrinsèquement bestiale - finit par prendre le dessus sur ce type d'amour hautement théorique et abstrait. Un peu à la façon naturaliste, le corps (souvent celui de l'homme !) finit par trahir les bonnes intentions d'un esprit pur à la recherche d'un idéal amoureux ${ }^{19}$. 
Enfin, j'aimerais terminer avec le besoin de liberté qui habite les personnages féminins de Rachilde. Toutes, à peu de romans près, revendiquent cette liberté que leur sexe - dit faible leur interdit socialement. À l'instar des célibataires hommes qui refusent le mariage, et donc le devoir de reproduction de l'espèce, ces célibataires rachildiennes aspirent à un autre destin (même si ce destin se termine généralement par leur mort - sorte de rétablissement de l'ordre confirmant ainsi la force de la normal sociale). Plusieurs romans de l'écrivaine présentent, à un certain moment, un chapitre sortant du lot, une sorte de digression en quelques paragraphes, ou bien un soliloque dénonçant la situation ingrate de la femme face au désir/plaisir de l'homme, rapidement assouvi. Le chapitre VII de Monsieur Vénus - véritable aparté aux propos revendicateurs à l'égard des femmes - l'explicite :

L'homme assis à sa propre droite sur les nuées d'un ciel imaginaire a relégué sa compagne au second rang dans l'échelle des êtres. En cela, l'instinct mâle a prévalu. Le rôle inférieur que sa conformation impose à la femme dans l'acte générateur, éveille évidemment une idée de joug et d'asservissement. L'homme possède, la femme subit. Les facultés passionnelles de celui-ci ne vont pas au-delà des limites de sa puissance physique. Quand la procréation a fait son œuvre, l'apaisement descend en lui. Rien ne survit au paroxysme sensuel. [...] Tout est dit pour l'homme, repu, brisé, anéanti, il s'écroule, et, pourtant avide d'étreintes, appelant d'autres caresses, évoquant de nouvelles joies, à ses côtés, la femme se prostitue aux conceptions paradisiaques. L'homme est matière, la volupté est femme, c'est l'éternelle inapaisée. (91-92)

Dans L'animale, Laure rejette d'emblée le mariage, sentant qu'elle n'y trouverait pas son compte, préférant sa liberté même si elle est incertaine et précaire : «Se marier ? Elle n'y tenait pas beaucoup, ayant bien deviné que le mariage n'assouvissait pas les créatures de sa trempe. Elle rêvait d'une autre vie» $(62 ; 70)$. Ayant «le dégoût de l'union, qui détruit $[\mathrm{s}] \mathrm{a}$ force » (La jongleuse, 49), Éliante, quant à elle, souhaite « être heureuse toute seule, les bras bien croisés sur [s]a poitrine, les cuisses jointes hermétiquement, avec le sourire des vierges qui communient» (167). Mary Barbe, dans La marquise de Sade, revendique sa liberté en s'opposant, dès sa nuit de noces, à son devoir de procréatrice pourvoyeuse d'héritier ${ }^{20}$, et ce, en menaçant son nouvel époux de l'empoisonner si ce dernier ne respectait pas ses volontés. Si Mary ne peut éviter le mariage (l'un des trois piliers [mariage/procréation/famille] des valeurs sociales bourgeoises), étant donné sa condition de femme à une époque où très peu de droits/moyens lui sont reconnus et mis à sa disposition, elle aspire tout de même à une certaine liberté. Pour ce qui est du désir de la femme, plusieurs célibataires rachildiennes critiquent cette 
inégalité du plaisir entre l'homme et la femme et la fugacité des sentiments amoureux de l'homme. Les femmes rachildiennes recherchent plutôt «l'infini du plaisir » (La jongleuse, 50), quitte à être seules avec un vase, telle Éliante.

Mais ce n'est pas le but, le plaisir ; c'est une manière d'être. Moi, je suis toujours... heureuse. Je voulais vous mener ici pour vous prouver que je n'ai pas besoin de la caresse humaine pour arriver au spasme... Il me suffit d'être... [...] car je porte en moi le secret de toutes les sciences en ne sachant qu'aimer. [...] Pour que ma chair s'émeuve [...], je n'ai pas besoin de chercher un sexe à l'objet de mon amour ! je suis humiliée parce qu'un homme intelligent pense tout de suite à...coucher avec moi... Demain vous ne m'aimeriez plus... si vous m'aimez si peu que cela. En effet, vous ne m'aimez pas, monsieur. Alors que prétendez-vous m'offrir ? Quelle confiance peut-on avoir dans cet homme qui passe ? Vous ne passerez pas chez moi... [...] Un frisson ? Ce n'est pas beaucoup pour celle qui est le frisson incarné ! Une flamme ? C'est trop peu pour une qui est toute la fournaise ! (49-50)

Encore une fois, c'est Éliante qui nous offre, de façon plus complète, une critique de la condition de la femme de l'époque et montre, tout comme Rachilde le dit dans son pamphlet ${ }^{21}$, que la femme a assimilé depuis fort longtemps sa condition d'asservissement, et ce, peu importe les progrès de cette soi-disant modernité :

Non, tu n'es pas le seul qui ose dire : la femme honnête est celle qui cède. Vous avez tous inventé cela dès votre berceau pour la plus grande commodité de vos futures alcôves, et vous l'avez tellement répété que les plus niaises le croient, aujourd'hui, s'étant enfin débarrassées de quelques préjugés divins. Elles naissent aussi en le croyant, et on entend même les jeunes filles charmantes déclarer, les poings en l'air, qu'elles cèderont, l'occasion offerte, pour s'assurer éternellement des droits qui ne s'acquièrent qu'avec une diabolique expérience ${ }^{22}$. (171)

J'aimerais finir ce cri de liberté féminin avec les propos de Rachilde elle-même tirés de son pamphlet qui résument bien ce qu'on trouve un peu partout dans son œuvre romanesque, comme si elle vivait à travers ses femmes de papier : «Par-dessus tout j'aime la liberté... surtout la mienne ! » (72)

Pour conclure, on reproche souvent à Rachilde son côté répétitif, certains vont encore plus loin en affirmant (à tort et de façon fort réductrice, à mon sens) qu'elle n'a cessé de réécrire encore et toujours la même œuvre, son Monsieur Vénus. Peut-être, au contraire, faut-il y voir là une superstructure, un fil d'Ariane qui mettrait en lumière une cohérence, une façon de concevoir ce que tous les personnages de Rachilde recherchent (ou tentent d'éviter) : l'amour. Participant à la mode fin-de-siècle du personnage célibataire masculin refusant l'amour normal (mariage/famille), mais parfois en décalage avec elle, grâce à ses personnages de femmes 
célibataires qui se marginalisent davantage encore, Rachilde nous offre un amour empreint de liberté, une liberté qui se veut un retour vers des temps primitifs. Prince, dans son ouvrage sur les célibataires fantastiques, montre comment ce personnage - tant réel que de papier - dérange par son inutilité sociale, puisqu'il n'y contribue en aucune manière. N'y a-t-il pas plus inutile, plus dérangeant pour une société qu'une femme qui refuse son rôle de perpétuation de l'espèce (et qui tente, tant bien que mal, d'acquérir une certaine indépendance avec - permettez-moi l'expression - les moyens du bord) ? Certes, je n'insinue pas ici que Rachilde était féministe - bien loin de là (elle l'a elle-même dit et redit à plusieurs occasions - son pamphlet à l'appui). Néanmoins, on ne peut nier l'existence de certaines phrases rebelles, en contradiction avec sa propre créatrice, prouvant par-ci par-là un goût criant pour la liberté d'être, d'agir et d'aimer peu importe le genre. Ces femmes parfois viriles, tantôt philosophes et, surtout, toujours étranges et en décalage sont, à mon sens, des preuves que chez Rachilde, le banquet est ouvert à toutes et à tous.

\section{Bibliographie}

Auriant. Souvenirs sur Madame Rachilde. Paris : À l'écart, 1989.

Baronian, Jean-Baptiste. « Rachilde ou l'amour monstre ». Magazine littéraire 288 (1991) : 4246.

Gauthier, Vicky. «La métamorphose du célibataire chez Rachilde ». Voix plurielles 9.2 (2012) : 105-115.

Hawthorne, Melanie. «Préface ». Monsieur Vénus, roman matérialiste. Rachilde. New York : MLA, coll. «MLA Texts and Translations », 2004.

Mayer, Regina B. Éros décadent : sexe et identité chez Rachilde. Paris : Champion, 2002.

Platon. Le banquet. Paris : Folio, coll. «Essais », 2008.

Prince, Nathalie. Les célibataires du fantastique : essai sur le personnage célibataire dans la littérature de la fin du XIX $X^{e}$ siècle. Paris : Harmattan, 2002.

Rachilde. La marquise de Sade. Paris : Mercure de France, 1981 [1887].

---. L'amazone rouge. Paris : Lemerre, 1932.

---. L'animale. Paris : Mercure de France, 1993 [1893].

---. Le grand saigneur. Paris : Flammarion, 1922.

---. La jongleuse. Paris : Des femmes, 1982 [1900].

---. Madame Adonis. Paris : Monnier, 1888. 
---. Madame de Lydone, assassin. Paris : Ferenczi, 1929.

---. Monsieur Vénus. New York : MLA, 2004 [1884].

---. Nono. Paris : Mercure de France, [1885] 1994.

---. Pourquoi je ne suis pas féministe. Paris : Éditions de France, coll. « Leurs raisons », 1928.

---. Refaire l'amour. Paris : Ferenczi, 1928.

Stead, Evanghélia. Le monstre, le singe et le foetus : tératogonie et décadence dans l'Europe finde-siècle. Genève : Droz, 2004.

$--$

\section{NOTES}

${ }^{1}$ Regina B. Mayer aborde brièvement la question de l'amour platonique dans son ouvrage Éros décadent : Sexe et identité chez Rachilde, et ce, dans trois œuvres de Rachilde (La fille inconnue, L'heure sexuelle et À mort), dont nous ne parlerons pas ici.

${ }^{2}$ Rappelons à cet effet que les œuvres de Rachilde sont nombreuses et débordent largement du dix-neuvième siècle puisqu'elle a vécu presque cent ans ; son dernier ouvrage est paru en 1947, ce qui peut expliquer le fait que bon nombre de ses personnages aient une position arrêtée par rapport à la modernité et ses avancées technologiques (électricité, aviation, voiture, etc.). Voir à ce propos mon article «La métamorphose du célibataire ».

${ }^{3}$ Pratiques toujours infécondes (souvent narcissiques et onanistes). Pour n'en nommer que quelques-unes : Éliante pratique l'autoérotisme avec un vase «très vieux, [qui] a des siècles » (La jongleuse, 46). Laure a des rapports érotiques avec son chat Lion. Raoule de Vénérande se travestit et bat son amant, Jacques Silvert, qu'elle drogue et habille en femme pour, ultimement, le faire assassiner et en faire un mannequin de cire pour son plaisir sexuel. Mary Barbe violente, mutile ses amants et en vient même à tuer pour son plaisir sadique.

${ }^{4}$ Jacques est comparé à Antinoüs (Voir Monsieur Vénus, 159).

${ }^{5}$ Cet endroit étrange du logis du personnage rachildien varie d'un roman à l'autre, mais est constamment présent : parfois il s'agit d'une serre (Refaire l'amour), d'une chambre à coucher/boudoir (Monsieur Vénus, La jongleuse, Madame Adonis), d'une salle de bains extérieure (Nono) ou même de la maison en entier (Madame Adonis, Madame de Lydone, assassin).

${ }^{6}$ L'auteure le répète également à la page 52 : «Le célibataire est celui qui voit sous la femme charmante la bête immonde ou le sexe monstrueux ».

${ }^{7}$ Aphrodite y est aussi présente et se voit tout aussi transformée (démonisée même) sous la plume décadente.

${ }^{8}$ La poétesse grecque Sapho est aussi présente dans certains textes de Rachilde, tels que dans Madame Adonis et Monsieur Vénus.

${ }^{9}$ Bien entendu, il y a de ces femmes mortifères chez Rachilde - qui ne vont pas nécessairement à l'encontre de la déesse Diane, puisqu'elle est considérée comme une déesse cruelle et vindicative. Pensons, par exemple, à ces hérö̈nes qui causent la mort, entre autres à Raoule de Vénérande dans Monsieur Vénus, à Mary Barbe dans $L a$ marquise de Sade. Il n'en demeure pas moins qu'elles promeuvent un discours empreint de liberté, dont il sera question plus loin dans cet article.

10 «De plus, [elle déclare qu'elle est] assez, EN ÉTANT, et [que si elle] pouvai[t] finir le monde avec [elle], [elle] le finirai $[\mathrm{t}] \gg$ (La marquise de Sade, 214).

${ }^{11}$ Ce roman - différent de ceux dont il est question ici - raconte plutôt la déchéance du personnage féminin qui incarne, pour son père, peintre renommé, la déesse Diane adolescente. Féa tombe enceinte de son violeur et perd, par le fait même, sa virginité qui l'élevait au niveau de la déesse.

${ }^{12}$ Qui plus est, dans la demeure de Marcelle Désambres, on trouve plusieurs trophées de chasse (Rachilde, Madame Adonis, 193) et on nous dit d'elle qu'elle « croquait le gibier et buvait le vin blanc comme un chasseur » (192).

${ }^{13}$ Chez la veuve Marcelle Désambres, il y a même une haine pour le sexe masculin : «Je n'ai jamais aimé les hommes, ils sont si bêtes et si brutaux. Mon mari m'en a dégoûtée pour le reste de ma vie » (Madame Adonis, 205). C'est pourquoi on trouve chez elle Sapho, à la fois en peinture et en sculpture. Le texte compare même Marcelle à ces œuvres d'art lorsqu'elle est décrite physiquement.

${ }^{14}$ Le nombril serait à cet effet la cicatrice, la marque de cette séparation antérieure. 
${ }^{15}$ Melanie C. Hawthorne le remarque aussi dans son édition annotée de Monsieur Vénus. Par ailleurs, le mythe de l'androgyne est aussi présent dans le roman Madame Adonis, dont la chute inattendue révèle que Marcelle Désambres et son frère Marcel sont, en réalité, une seule et même personne (il s'agissait au final de Marcelle qui avait réussi à séduire, chacun leur tour, le couple Louis et Louise Bartau). Certains indices sont laissés çà et là dans le roman, notamment lors des descriptions physiques de Marcel(le), tantôt féminines, tantôt masculines. Elle constitue à elle seule l'androgyne (homme et femme à la fois), contrairement à Monsieur Vénus, où Raoule et Jacques sont ce tout androgyne.

16 «Voilà donc quelle est la droite voie qu'il faut suivre dans le domaine des choses de l'amour [...] : c'est, en prenant son point de départ dans les beautés d'ici-bas, avec, pour but, cette beauté surnaturelle, de s'élever sans arrêt, comme au moyen d'échelons : partant d'un seul beau corps de s'élever à deux, et, partant de deux à s'élever à la beauté des corps universellement; puis, partant des beaux corps, de s'élever aux belles occupations, et partant des occupations, de s'élever aux belles sciences jusqu'à ce que, partant des sciences, on parvienne, pour finir, à cette science sublime, qui n'est science de rien d'autre que de ce beau surnaturel tout seul, et qu'ainsi, à la fin, on connaisse, isolément, l'essence même du beau » (Platon 132-133).

${ }^{17}$ Ce que fera d'ailleurs Yves de Pontcroix avec ses comportements amoureux à caractère vampirique sanguinaire et son étrange vocabulaire où ce dernier remplace amour par mort : « Cet homme [Pontcroix] n'avait donc pas de cœur qu'il remplaçait le mot amour par mort »; «Marie, je désire votre vie, votre sang, votre admirable santé, votre adorable santé. »; «[...] cette étrange façon de concevoir l'amour d'outre-tombe »; «[...] je vous [Marie] aime assez, moi, pour ne pas vous imposer mon amour qui ne ressemble pas tout à fait à l'autre » (Le grand saigneur, 104, 105, 178 et 273). On trouve la même chose chez Monsieur Vénus dans le fameux chapitre VII, qui fut censuré lors de sa réédition en 1889, et qui serait, semble-t-il, attribuable à un certain Talman (dans une lettre au critique et ami Auriant, Rachilde dit que le chapitre VII « fut enlevé d'accord avec l'auteur et l'éditeur. » Elle écrit ensuite que « ce chapitre est vraiment le comble de tous les ridicules... À retenir surtout la première phrase : l'homme assis à sa propre droite. Cette phrase faisait rire Alfred Vallette quoiqu'il ne pût pas sentir son auteur, lequel était un assez joli garçon genre séducteur pour jeunes filles du meilleur monde. » (Lettre du 10 octobre 1938 de Rachilde à Auriant, Souvenirs sur Madame Rachilde, 29) : «La volupté est femme. Dans l'irradiation d'une aurore vengeresse, la femme entreverra pour l'homme la possibilité d'une fabuleuse chute. Elle inventera des caresses, trouvera de nouvelles preuves aux nouveaux transports d'un nouvel amour et Raoule de Vénérande possédera Jacques Silvert... » (94).

${ }^{18}$ Cette idée que l'Amour élève l'individu vers le Beau et le Bon est tout à fait dans la ligne de pensée platonicienne. ${ }^{19}$ À juste titre, Éliante se refuse tout au long du texte à Léon (qui souhaite ardemment devenir son amant, c'est-àdire de la posséder) et choisit ultimement de mettre fin à ses jours.

${ }^{20}$ Dans Refaire l'amour, la maternité et ses dommages - parfois mortels - sur la femme sont aussi relatés. Montarès apprend la mort de Bouchette, une jolie fille qui a posé pour lui le temps d'un dessin : « Or, cette bouchette-là est morte en couches, déformée, abîmée, roulée aux abîmes de la grande marâtre, notre mère, la Terre, qui exige de nous la procréation...ou la mort » (253).

${ }^{21}$ Étant donné le peu de temps imparti, je ne fais que relever les pages concernées, 50 et 53, du pamphlet de Rachilde.

${ }^{22}$ Autre occurrence par rapport à l'asservissement de la femme dans La jongleuse : «Je sais que ce temps-ci n'est pas fertile en grâce... j'ai peur que demain la grâce de la femme... soit reconnue d'utilité publique et se spécialise au point de devenir l'article banal, un objet de bazar à treize et que l'on trouve des catégories de femmes tendres ou amusantes des milliers d'exemplaires comme les confections des grands magasins... de nouveauté où c'est toujours la même chose » (167). 